\title{
ON THE DENJOY GONJEGTURE
}

\author{
JAMES A. JENKINS
}

1. In recent years many of the properties of regular functions have been shown to extend to quasiconformal mappings. (The latter term is here understood in the sense defined in (5).) This is particularly true of those results which can be proved by use of the method of the extremal metric. It is rather strange, then, that a result which constitutes one of the first notable applications of this method has not been so extended (at least to the author's knowledge). Here we refer to the proof of the Denjoy conjecture given by Ahlfors (1). The reason for this may be that Ahlfors' proof uses in an essential manner the principle of majoration for harmonic functions and this part of the proof does not extend readily to quasiconformal mappings. Not long afterwards another proof was published by Beurling (2). The same situation appears to hold for this proof although in some ways it is probably closest in spirit to the one given below. Later proofs are either rather technical modifications of Ahlfors' proof or follow that of Carleman (3) and so contain features rendering them unsuitable for generalization to quasiconformal mappings.

In this paper we give the extension of the Denjoy conjecture to quasiconformal mappings. Our proof uses the principle of harmonic majoration only in proving one self-contained lemma and otherwise operates exclusively with the method of the extremal metric.

2. A principal role will be played by a geometrical configuration which we will call a triad. This consists of a simply-connected domain of hyperbolic type, say $D$, an open boundary arc of $D$ (in the natural sense of boundary correspondence), say $\gamma$, and a distinguished interior point $P$ of $D$. We denote in the usual way by $\omega(P, \gamma, D)$ the harmonic measure of $\gamma$ taken at $P$ with respect to the domain $D$. Also we will use the notation $\gamma^{*}$ for the closed boundary arc of $D$ complementary to $\gamma$. We denote by $m(P, \gamma, D)$ the module $(4,6)$ of the class of (locally rectifiable) open arcs lying in $D-\{P\}$, running from $\gamma$ back to $\gamma$ and separating $P$ from $\gamma^{*}$. It is well known that there is a monotone increasing function from the interval $[0,1)$ to the half-infinite interval $[0, \infty)$ which relates $\omega(P, \gamma, D)$ to $m(P, \gamma, D)$. For real numbers $a$ and $b, a<b$, we denote by $S(a, b)$ the strip in the $(u, v)$-plane defined by

$$
a<u<b
$$

by $g(a)$ its boundary arc $u=a$ and by $g(b)$ its boundary $\operatorname{arc} u=b$.

Received October 9, 1957. Research supported in part by the Mathematics Section, National Science Foundation through the University of Notre Dame and partially supported by the Office of Ordnance Research, U. S. Army under contract No. DA-36-034-ORD-2453. 
Lemma 1. Consider a triad consisting of a Jordan domain $D$ in the z-plane, an open boundary arc $\gamma$ of $D$ and an interior point $P$ of $D$. Let $f$ be a mapping quasiconformal on $\bar{D}$, of maximal dilation (5) $K$, which satisfies

$$
\begin{aligned}
& |f(z)| \leqslant A, \quad z \in \gamma \\
& |f(z)| \leqslant B, \quad z \in \gamma^{*}
\end{aligned}
$$

with $0<A<B$. Then

$$
m(P, \gamma, D) \leqslant K m(\log |f(P)|, g(\log A), S(\log A, \log B))
$$

where the logarithms in each case denote the principal value.

There exists a triad consisting of a Jordan domain $D^{\prime}$ in the $z^{\prime}$-plane, a boundary arc $\gamma^{\prime}$ of $D^{\prime}$ and an interior point $P^{\prime}$ of $D^{\prime}$ related to the given triad by a univalent mapping $z=\phi\left(z^{\prime}\right)$, quasiconformal on $\bar{D}^{\prime}$ and of maximal dilation $K$ such that the function

$$
g\left(z^{\prime}\right)=f\left(\phi\left(z^{\prime}\right)\right)
$$

is regular on $\bar{D}^{\prime}$. Then we have by the two constants theorem

$$
\log \left|g\left(P^{\prime}\right)\right| \leqslant(\log A) \omega\left(P^{\prime}, \gamma^{\prime}, D^{\prime}\right)+(\log B)\left(1-\omega\left(P^{\prime}, \gamma^{\prime}, D^{\prime}\right)\right)
$$

that is

$$
\begin{aligned}
\omega\left(P^{\prime}, \gamma^{\prime}, D^{\prime}\right) & \leqslant \frac{\log B-\log \left|g\left(P^{\prime}\right)\right|}{\log B-\log A} \\
& \leqslant \omega(\log |f(P)|, g(\log A), S(\log A, \log B)) .
\end{aligned}
$$

In view of the monotonic correspondence between the harmonic measures and the modules of triads we have

$$
m\left(P^{\prime}, \gamma^{\prime}, D^{\prime}\right) \leqslant m(\log |f(P)|, g(\log A), S(\log A, \log B)) .
$$

On the other hand we have evidently

$$
m(P, \gamma, D) \leqslant K m\left(P^{\prime}, \gamma^{\prime}, D^{\prime}\right)
$$

from which inequality (1) follows at once.

While we have used harmonic majoration in the proof of this result its statement involves only modules. It would be very interesting to have a proof of Lemma 1 purely by the method of the extremal metric.

Lemma 2. If $l<\lambda<L$ are real numbers then for $l, \lambda$ fixed and $L$ tending to infinity

$$
m(\lambda, g(l), S(l, L)) \leqslant \frac{1}{\pi} \log L+O(1) .
$$

Let $\Gamma$ be the class of (locally rectifiable) open arcs lying in $S(l, L)-\{\lambda\}$, running from $g(l)$ back to $g(l)$ and separating $\lambda$ from $g(L)$. To provide an admissible metric in $S(l, L)$ for the module problem for $\Gamma$ we define first the square $\Sigma(l, L)$ 


$$
l<u<L,-\frac{L-l}{2}<v<\frac{L-l}{2} .
$$

Let $E(l, \lambda, L)$ be the half of the open elliptical disc to the right of the line $u=l$ which is bounded by the ellipse with centre at $l$, foci at $\lambda, 2 l-\lambda$ and passing through the point $\left(L, \frac{1}{2}(L-l)\right)$. For the semi-axis major $a$ of this ellipse we have

$$
\left(a^{2}-(\lambda-l)^{2}\right)(L-l)^{2}+\frac{1}{4}(L-l)^{2} a^{2}=a^{4}-a^{2}(\lambda-l)^{2}
$$

so that for $L$ large enough

$$
a \leqslant \frac{3}{2} L
$$

Let now $\rho^{*}(w)|d w|$ be the extremal metric in the module problem for the family of curves $\Xi$ lying in $E(l, \lambda, L)$, running from the minor axis back to the minor axis and separating $\lambda$ from the elliptical boundary arc. It is well known that this family has module

$$
m(\Xi)=\frac{1}{\pi} \log \mathbf{P}
$$

where $\mathbf{P}$ is the larger root of

$$
\mathrm{P}+\mathrm{P}^{-1}=2(\lambda-l)^{-1} a .
$$

Evidently

$$
m(\Xi) \leqslant \frac{1}{\pi} \log \left[2(\lambda-l)^{-1} a\right] \leqslant \frac{1}{\pi} \log L+O(1) .
$$

Now we regard the metric $\rho(w)|d w|$ defined by

$$
\begin{aligned}
\rho(w) & =0, & w \in S(l, L)-\Sigma(l, L) \\
& =\max \left(\rho^{*}(w),(L-l)^{-1}\right), & w \in \Sigma(l, L) .
\end{aligned}
$$

Considering $\Gamma$ as composed of the three classes of curves which meet both, just one, or neither of the horizontal sides of $\Sigma(l, L)$ and recalling the symmetry of the metric $\rho(w)|d w|$ in the real axis we see that this metric is admissible in the module problem for $\Gamma$. In it the area of $S(l, L)$ is less than $m(\Xi)+1$. Thus

$$
m(\lambda, g(l), S(l, L)) \leqslant \frac{1}{\pi} \log L+O(1)
$$

as stated.

3. The concepts of an integral quasiconformal mapping and its maximum modulus are evident generalizations of similar ones for regular functions. So are the concept of an asymptotic value of such a function and the manner of counting distinct asymptotic values. We are now ready to state our principal result. 
THEOREM. If the integral quasiconformal mapping $f(z)$ of maximal dilation $K$ has $n$ distinct asymptotic values and $M(r)$ denotes the maximum modulus. of $f(z)$ for $|z|=r$, then

$$
\varliminf_{n \rightarrow \infty} M(r) r^{-n / 2 K}>0 .
$$

The standard reduction of the problem enables us to consider the following: situation. There exist a circle $|z|=R_{0}\left(R_{0}>0\right)$ and $n$ non-intersecting open $\operatorname{arcs} A_{j}, j=1, \ldots, n$, running in $|z|>R_{0}$ from this circle to the point at infinity and dividing $|z|>R_{0}$ into $n$ domains $D_{j}, j=1, \ldots, n$. Further, there is a positive constant $\alpha$ such that on $|z|=R_{0}$ and on the $A_{j}, j=1, \ldots, n$. we have

$$
|f(z)| \leqslant \alpha .
$$

Finally, in each domain $D_{\jmath}, j=1, \ldots, n$, there is a point $P_{j}, j=1, \ldots, n$, of modulus $\beta_{j}$ for which

$$
\left|f\left(P_{j}\right)\right|>\alpha .
$$

Let

$$
\beta=\max _{j=1, \ldots, n} \beta_{j} .
$$

Then for $R>\beta$ there is on the circle $|z|=R$ a cross cut $B_{j}(R)$ in $D_{j}$ separating. the boundary arc of $D_{j}$ on $|z|=R_{0}$ and the point $P_{j}$ from the boundary point of $D_{j}$ at infinity. Let the component of $D_{j}-B_{j}(R)$ containing $P_{j}$ be denoted by $D_{j}(R)$. Let the open boundary arc of $D_{j}(R)$ complementary to the closure of $B_{j}(R)$ be denoted by $C_{j}(R)$. Let $\rho_{j}(z, R)|d z|$ be the extremal metric in the module problem defining $m\left(P_{j}, C_{j}(R), D_{j}(R)\right), j=1, \ldots, n$.

We denote by $K(\beta, R)$ the circular ring

$$
\beta<|z|<R \text {. }
$$

In $K(\beta, R)$ we define the metric $\rho(z, R)|d z|$ by

$$
\begin{array}{lll}
\rho(z, R)=\rho_{j}(z, R), & & z \in K(\beta, R) \cap D_{,}(R), j=1, \ldots, n, \\
\rho(z, R)=0, & & \text { elsewhere in } K(\beta, R) .
\end{array}
$$

Then if the rectifiable Jordan curve $c$ separates the boundary components of $K(\beta, R)$ we see at once

$$
\int_{c} \rho(z, R)|d z| \geqslant n
$$

so that

$$
\frac{1}{2 \pi} n^{2} \log \frac{R}{\beta} \leqslant \sum_{j=1}^{n} m\left(P_{j}, C_{j}(R), D_{j}(R)\right) .
$$

By Lemma 1, since

$$
\begin{array}{ll}
|f(z)| \leqslant \alpha & \text { on } C_{j}(R), \\
|f(z)| \leqslant M(R) & \text { on } B_{j}(R),
\end{array}
$$

we have 


$$
\begin{aligned}
& \sum_{i=1}^{n} m\left(P_{j}, C_{j}(R), D_{j}(R)\right) \\
& \leqslant K \sum_{j=1}^{n} m\left(\log \left|f\left(P_{j}\right)\right|, g(\log \alpha), S(\log \alpha, \log M(R))\right) .
\end{aligned}
$$

Moreover, by Lemma 2,

(4) $\sum_{j=1}^{n} m\left(\log \left|f\left(P_{j}\right)\right|, g(\log \alpha), S(\log \alpha, \log M(R))\right) \leqslant \frac{n}{\pi} \log \log M(R)+O(1)$.

Combining (2), (3), and (4) we find

$$
\frac{1}{2 \pi} n^{2} \log R+O(1) \leqslant K \frac{n}{\pi} \log \log M(R)
$$

so that

$$
\log M(R) \geqslant S R^{n / 2 K}
$$

for a suitable positive constant $S$ and $R$ large enough. This proves our theorem.

Our resullt can be restated in a perhaps more familiar but weaker form in saying that an integral quasiconformal mapping of order $\rho$ (the extension of this notion from integral functions is also immediate) which has maximal dilation $K$ has at most $\left[2 K_{\rho}\right]$ asymptotic values. An evident modification of the corresponding example for integral functions shows this result to be best possible.

\section{REFERENCES}

1. Lars V. Ahlfors, Untersuchungen zur Theorie der konformen Abbildung und der ganzen Funktionen, Acta Societatis Scientiarum Fennicae, Nova Series A, Opera Phys.-math., 1, (1930), no. 9, 1-40.

2. A. Beurling, Etudes sur un problème de majoration, thesis (Upsala, 1933).

3. T. Carleman, Sur une inégalité différentielle dans la théorie des fonctions analytiques, C. R. Acad. Sci. Paris, 196 (1933), 995-997.

4. James A. Jenkins, Symmetrization results for some conformal invariants, Amer. J. Math., 75 (1953), 510-522.

5. - On quasiconformal mappings, J. Rat. Mech. and Anal., 5 (1956), 343-352.

6. - Univalent functions and conformal mapping, No. 18, Ergebnisse der Mathematik, Springer-Verlag (to appear).

University of Notre Dame

and

Institute of Advanced Study 